\title{
Single layer narrow bandwidth angle-insensitive guided-mode resonance bandstop filters
}

\author{
Gaige Zheng $^{\mathrm{a}, \mathrm{b}^{*}}$, Xiujuan Zou ${ }^{\mathrm{a}}$, Linhua Xu ${ }^{\mathrm{a}}$, Jicheng Wang ${ }^{\mathrm{c}}$ \\ ${ }^{a}$ School of Physics and Optoelectronic Engineering, Nanjing University of \\ Information Science \& Technology, Nanjing 210044, China \\ ${ }^{\mathrm{b}}$ Jiangsu Collaborative Innovation Center on Atmospheric Environment and \\ Equipment Technology (CICAEET), Nanjing University of Information Science \& \\ Technology, Nanjing 210044, China \\ ${ }^{\mathrm{c}}$ School of Science, Optoelectronic Engineering and Technology Research \\ Center,Jiangnan University, Wuxi 214122, China \\ *Corresponding author: jsnanophotonics@yahoo.com
}

\begin{abstract}
:
Ultra-narrow linewidth, angle insensitive guide-mode resonance filter (GMRF) has been designed. The aimed performance on the behavior of the filter with respect to the angular divergence of the incident beam was considered. Results show that high reflection (more than 99.9\%) can be obtained at the resonance wavelength, the full-width at half maximum (FWHM ) of TE- polarized light is only $1.1 \times 10^{-3} \mu \mathrm{m}$, and its sideband reflectance from 0.565 to $0.625 \mu \mathrm{m}$ is less than $1 \%$. In contrast to the ordinary GMRF, where the location of the resonances is incident angle dependent, it is demonstrated that the maximum peaks of the reflected spectrum gradually decreases with respect to the incident angle, but the reflectance peak position does not change.

Key words: Guided mode resonance (GMR), bandstop filter, Finite Difference Time-Domain (FDTD) method
\end{abstract}




\section{Introduction}

A guided mode resonant (GMR) grating filter is a structure composed of a stack of thin dielectric layers, a grating is usually engraved which can support leaky guided modes and lead to resonance peaks in reflection and dips in transmission spectra [1-5]. GMR effect occurs when the evanescent diffraction orders coupled into the waveguide mode and propagate in waveguide layer at appropriate optical parameters such as wavelength, angle and state of polarization of incident light [3-5]. Although GMR filters have been extensively studied and designed, together with the expansion of application areas of the GMR filter, there are continuously new requirements for the filter performances to meet practical needs, such as narrower band, larger angular tolerance, oblique incidence, polarization independence, and so on. Among these, the independence of the filter characteristics with respect to the incident angle of the incident beam are crucial properties that are often requested in applications.

The angular sensitivity of GMRF is well known, the coupling of the evanescent diffracted orders to the leaky mode of the waveguide will result in the splitting of the resonance at non-normal incidence. The sensitivity of the peak spectral location to the angle of incidence can be used effectively to tune the central wavelength of the filter to the desired value. However, this sensitivity can also be a major detriment for practical application. Many research works have been done to avoid this kind of limitation in the past years [6,9-11]. Quite recently, a polymer-dispersed liquid crystal layer was introduced to compensate the shift of resonant wavelength through applying

proper voltage by B. L. Xu et al.[11], and a maximum angular compensation range of 
about $\pm 4.2^{\circ}$ was obtained while maintaining high performance of the spectral response. Multilayer waveguide-grating stacks support multiple resonant modes which limits the filter's free spectral range. It is of great interest to find waveguide gratings with fewer layers that nevertheless exhibit low reflection in the spectral region outside the resonance. Alasaarela et. al. reported a single layer GMRFs with the non-polarizing effect at normal incidence [12].

In this paper, we report a simpler design and show that properly designed 1D GMRFs with single grating layer on gradient-index substrate may exhibit the ultra-narrow linewidth and angle insensitive properties at the same time. The Finite Difference Time-Domain (FDTD) method is used to calculate the reflectivity spectra and optimize the structure parameters. The stop band of the reflection-type angle-independent filter can also be adjusted by varying the structure geometry. The angle-independent and ultra-narrow bandwidth features make the designed filter more flexible than either guided-mode resonance (GMR) dielectric filter [13-15] or plasmonic filter $[16,17]$.

\section{Numerical results with analysis and discussions}

Figure 1 shows an illustration of the proposed angle-independent GMRF with geometric parameters as follows: $d_{\mathrm{g}}=110 \mathrm{~nm}, d_{\mathrm{s}}=1000 \mathrm{~nm}, \Lambda=340 \mathrm{~nm}, f=0.2, n_{\mathrm{c}}=1.0$ and $n_{\mathrm{g}}=1.97$. The incident light is TE-polarized (i.e., electric field parallel to the $z$ axis) with varied incident angles in $x-y$ plane. The refractive index variation throughout the physical thickness of the substrate layer is described by:

$$
n(y)=\sqrt{n_{0}^{2}+n_{p}^{2} \mathrm{e}^{-\alpha y}-n_{p}^{2}}
$$


where $n_{0}, n_{\mathrm{p}}$ and $\alpha$ are chosen as $2.02,0.45836$ and $2.303 \times 10^{-6} / \mu \mathrm{m}$, respectively. Figure 2 shows the refractive index distribution of the graded-index layer in the $y$-axis direction. It is usually very difficult to fabricate an ideal GMRF with the same parameters as the designed one [18]. GMRF constructed with single-layer periodic waveguides can be used to reduce the fabrication error, the grating layer servers as the waveguide layer at the same time.

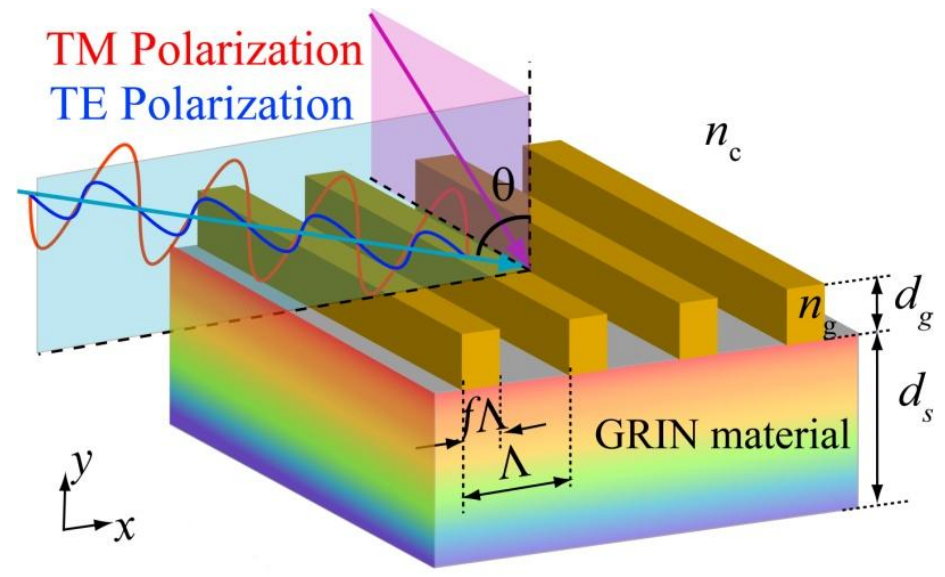

Fig. 1. Structure of single-layer GMR grating with gradient refractive index (GRIN) substrate.

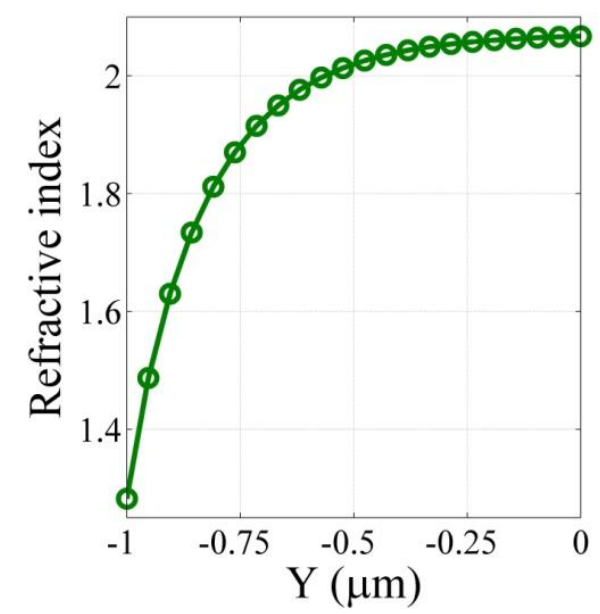

Fig.2. Refractive index distribution of the graded-index substrate along the $y$-axis.

Figure 3 illustrates the spectral responses of the GMRF for a normal-incidence TE-polarized wave. As can be seen, good spectral distributions with low sideband reflection and narrow-band width can be obtained. The GMR filter has almost $100 \%$ 
reflection at the resonant wavelength of $0.592 \mu \mathrm{m}$, with a spectral full width at half-maximum (FWHM) of $1.1 \times 10^{-3} \mu \mathrm{m}$, and its sideband reflectance from 0.565 to $0.625 \mu \mathrm{m}$ is less than $1 \%$. In order to investigate the origin of the narrow peak, we analyze the electric field distribution under resonance which is shown in Fig. 4 (a), where the amplitude magnitude $|\mathrm{E}|$ of the electric field normalized with respect to the incident over one grating period is shown. The electric field enhancement inside the substrate as resulting from the multiple interference of the energy coupled to the substrate from every grating period. The further the coupled waves propagate, the more coupled waves participate in the multiple interference and the bigger the maximum of the electric field [19]. Fig.4 (b) is the electric field distribution off resonance with wavelength of $0.58 \mu \mathrm{m}$, no electric field enhancement can be observed in this case.

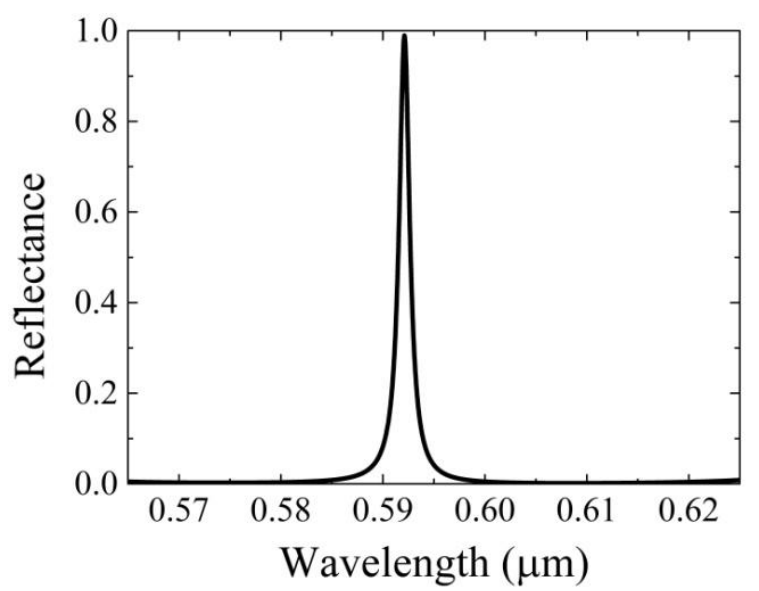

Fig.3. Reflection spectra under normal TE wave incidence with the parameters of the structure specified as $d_{\mathrm{g}}=110 \mathrm{~nm}, d_{\mathrm{s}}=1000 \mathrm{~nm}, \Lambda=340 \mathrm{~nm}, f=0.2, n_{\mathrm{c}}=1.0$ and $n_{\mathrm{g}}=1.97$.

The GMR filter with GRIN substrate has the desirable characteristics of narrow bandwidth and low sideband reflection. The resonant peak value of GMRF is susceptible to the fabrication errors of its parameters, such as the period, the groove 
depth, the thickness of a guided-wave layer, and the refraction indexes of materials, and so on. We designed structures with different substrate thickness while keeping all other parameters constant to allow for more easier fabrication. As shown in Fig. 5, the various thicknesses of the substrate have a distinct effect on the reflection spectrum of the structure. Keeping all other parameters the same, the FWHM at the resonant wavelength decreases with an increase in the thickness of the GRIN substrate. When $d_{\mathrm{s}} \geq 1 \mu \mathrm{m}$, the reflection curve will not change any more. The mode associated with the resonance will become less localized in the waveguide layer, which results in a drop of the coupling efficiency between the incident optical wave and the quasi-guided mode. When the thickness of the substrate is adequate, the quasi-guided mode will not escape from the waveguide layer, and thus a high efficiency GMR occurs [20].
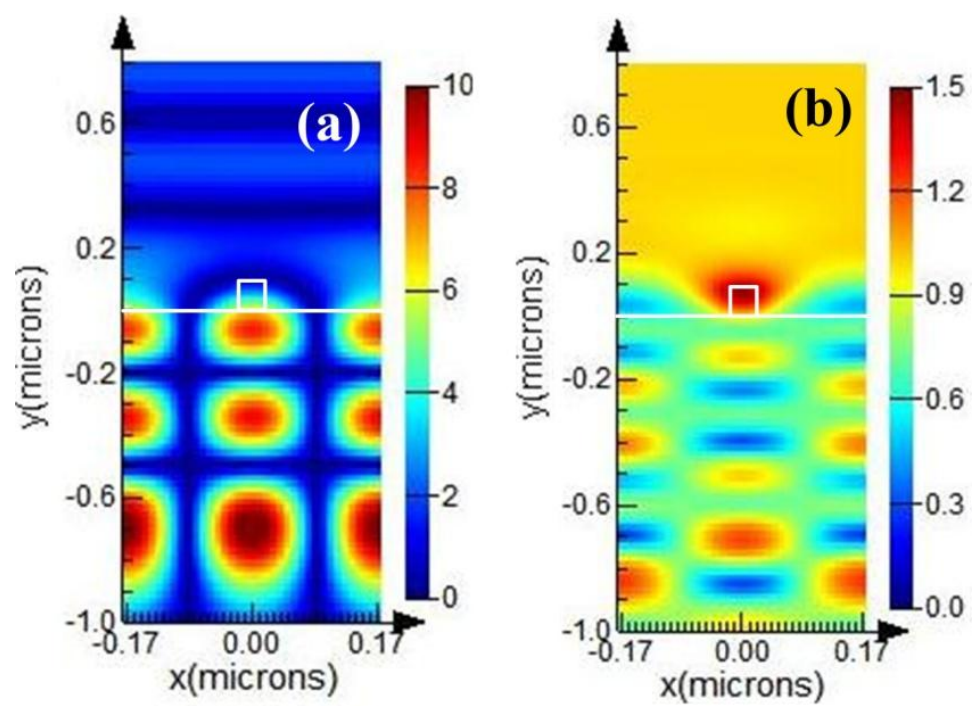

Fig.4. Electric field distribution profile at the resonant (a) and non-resonant wavelengths. White lines indicate the interfaces of the different layers. 


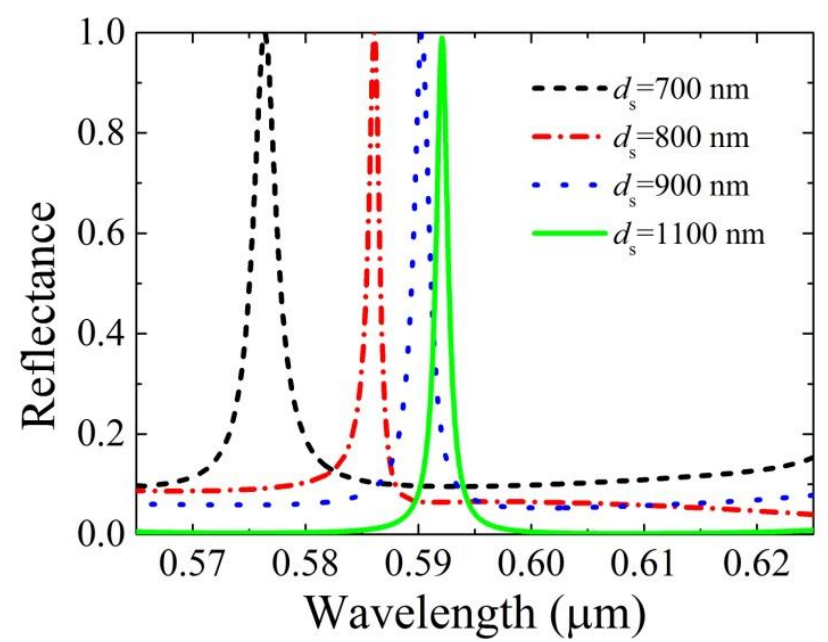

Fig.5. Reflection responses of the GMRF for various thicknesses of the substrate. The remaining parameters are the same as in Fig.3.

Though the various GMRFs have been examined for narrow or broad bandwidth optical filter properties, the resonance coupling is known to be very sensitive to incident angle of light, which makes the GMR filter difficult to use for certain application like color filter. Multilayer films offer an alternative solution to obtain color filters with narrow bandwidth. However, the multilayer filter requires a large number of dielectric layers and the loss of efficiency by light absorption makes the filter difficult to manufacture [21]. We also investigate the angular sensitivity of the designed GMRF. Fig. 6 shows the calculated reflectance spectra with three different incident angles. Although the maximum peaks gradually decreases with respect to the incident angle, the reflectance peak position does not change. We expect this GMR filter can be used as a reflective color filter for various display applications. The GMRF for the oblique incidence also exhibits electric field enhancement which can be seen from Fig.7, but this effect becomes less significant with the increasing angle in contrast with the normal case, because the structure is not at strong resonance. 


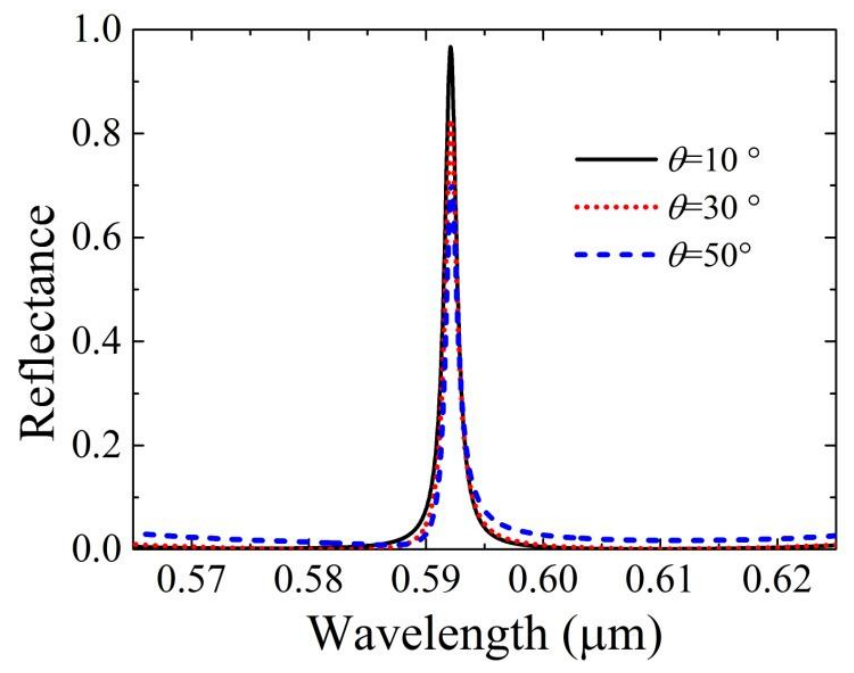

Fig.6. Comparisons of reflectance curves with respect to different incident angles.
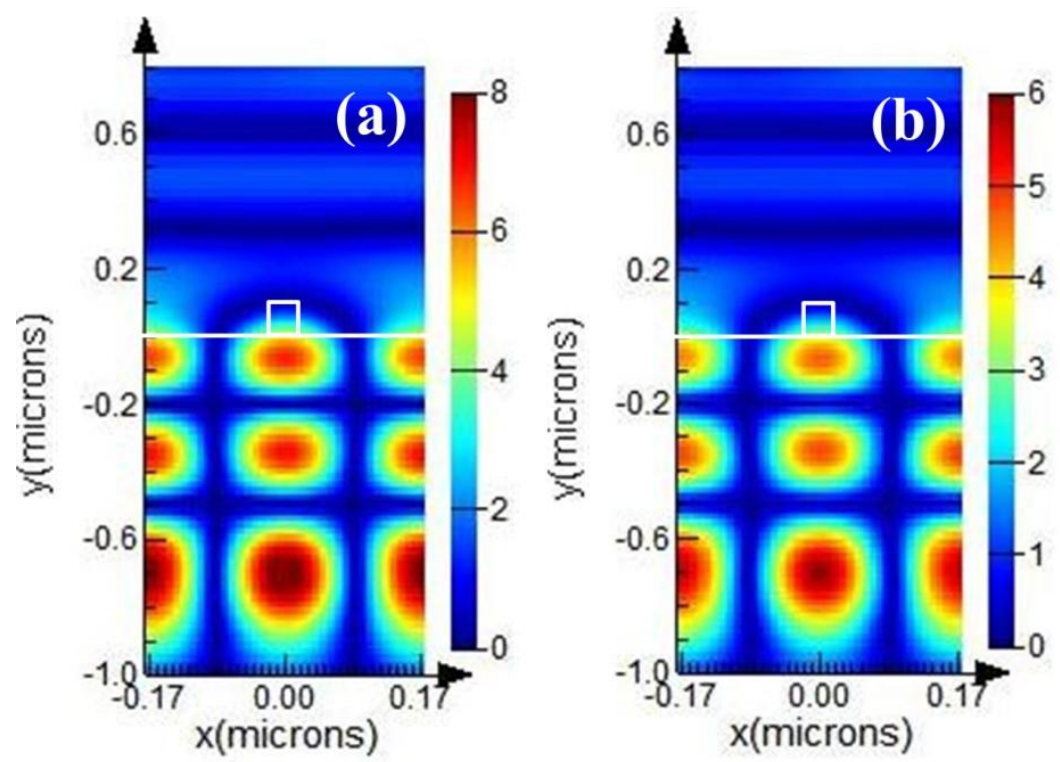

Fig.7. Electric field distribution profile at the resonant wavelength for oblique TE-polarized wave, the incident angles are $10^{\circ}$ (a) and $30^{\circ}$, respectively. White lines indicate the interfaces of the different layers.

\section{Summary}

GMR phenomena occurs when the evanescent orders of a diffraction grating are coupled to the waveguide modes and propagate out at given optical parameters such as wavelength, angle, and state of polarization of incident light. The outcoupling field from a waveguide is, in general, incident angle sensitive. Angle insensitive grating structures with high diffraction efficiency are highly required in practical applications. 
We have demonstrated a GMFR with grating structures placed on GRIN substrate. The angle-insensitive property with narrow bandwidth and low sideband reflection for TE-polarized wave has been verified.

\section{Acknowledgement}

This work was supported by the National Science Foundation of China (NSFC) (61203211, 41675154), the Six Major Talent Peak expert of Jiangsu Province (2015-XXRJ-014), and the Natural Science Foundation of the Jiangsu Province (BK20141483).

\section{References}

1. E. Popov, L. Mashev, and D. Maystre, Opt. Acta 33, 607 (1986).

2. S. S. Wang and R. Magnusson, Appl. Opt. 32, 2606 (1993).

3. D. Lacour, G. Granet, J.-P. Plumey, and A. Mure-Ravaud, J. Opt. Soc. Am. A 20, $1546(2003)$

4. G. Niederer, W. Nakagawa, H. P. Herzig, and H. Thiele, Opt. Express 13, 2196 (2005).

5. D. W. Peters, R. R. Boye, J. R. Wendt, R. A. Kellogg, S. A. Kemme, T. R. Carter, and S. Samora, Opt. Lett. 35, 3201 (2010).

6. A.-L. Fehrembach and A. Sentenac, Appl. Phys. Lett. 86, 121105 (2005).

7. X. Fu, K. Yi, J. Shao, and Z. Fan, Opt. Lett. 34, 124 (2009).

8. T. Alasaarela, D. Zheng, L. Huang, A. Priimagi, B. Bai, A. Tervonen, S. Honkanen, M. Kuittinen, and J. Turunen, Opt. Lett. 36, 2411 (2011)

9. F. Lemarchand, A. Sentenac, and H. Giovannini, Opt. Lett. 23, 1149 (1998).

10. X. Buet, E. Daran, D. Belharet, F. Lozes-Dupuy, A. Monmayrant, and O. 
Gauthier-Lafaye, Opt. Express 20, 9322 (2012).

11. B. L. Xu, D. W. Zhang, Y.S. Huang, Q. Wang, B. C. Li, and D. H. Zhang, IEEE Photon. Technol. Lett. 26, 231 (2014).

12. Tapani Alasaarela,Dandan Zheng, Lingling Huang, Arri Priimagi, Benfeng Bai, Ari Tervonen, Seppo Honkanen, Markku Kuittinen, and Jari Turunen, Opt. Lett. 36, $2411(2011)$.

13. D. L. Brundrett, E. N. Glytsis, and T. K. Gaylord, Opt. Lett. 23, 700 (1998).

14. F. Lemarchand, A. Sentenac, and H. Giovannini, Opt. Lett. 23, 1149 (1998).

15. A. L. Fehrembach, A. Talneau, O. Boyko, F. Lemarchand, and A. Sentenac, Opt. Lett. 32, 2269 (2007).

16. Z. Wu, J. W. Haus, Q. Zhan, and R. L. Nelson, Plasmonics 3, 103 (2008).

17. C. M. Wang, Y. C. Chang, M. W. Tsai, Y. H. Ye, C. Y. Chen, Y. W. Jiang, S. C. Lee, and D. P. Tsai, IEEE Photonics Technol. Lett. 20, 1103 (2008).

18. Q.Wang, D.W.Zhang, H.b.Heet.al., Opt. Lett. 34, 70(2009).

19. Tianyu Sun „JianyongMa, Jianpeng Wang, Yunxia Jin, Hongbo He, Jianda Shao and Zhengxiu Fan, J. Opt. A: Pure Appl. Opt. 10, 125003 (2008).

20. G. Niederer, H. P. Herzig, J. Shamir, H. Thiele, M. Schnieper, and C. Zschokke, Appl. Opt. 43, 1683 (2004).

21. S. Tibuleac and R. Magnusson, J. Opt. Soc. Am. A Opt. Image Sci. Vis 14, 1617 (1997). 